Supporting Information

Environmental Science \& Technology

\title{
Evaluation of the Intrinsic Photocatalytic Oxidation Kinetics of Indoor Air Pollutants
}

Ignasi SALVADÓ-ESTIVILL, David M. HARGREAVES ${ }^{\dagger}$ and Gianluca LI PUMA*

Photocatalysis \& Photoreaction Engineering, School of Chemical, Environmental and Mining Engineering, The University of Nottingham, University Park, Nottingham NG7 2RD, United Kingdom.

${ }^{\dagger}$ School of Civil Engineering, The University of Nottingham.

RECEIVED DATE (to be automatically inserted after your manuscript is accepted if required according to the journal that you are submitting your paper to)

* Corresponding author: Tel: +44 (0) 115 9514170; Fax: +44 (0) 115 9514115; Email: gianluca.li.puma@nottingham.ac.uk 


\section{Experimental Validation of LSSE Model}

Figure S1 shows the lamp arrangement in the reactor and coordinates of LSSE model. Figure S2 shows the fitting of the LSSE model to radiometric measurements of the radiation intensity at different positions on the catalytic plate, in the presence and in the absence of the borosilicate glass reactor cover. The net-radiation method (Siegel, R.; Howell, J. R., Thermal Radiation Heat Transfer. 4th ed.; Taylor \& Francis: New York, 2002) was used to calculate the radiation intensity on the catalyst in the presence of the borosilicate glass which absorbs and reflect radiation. The refractive index of borosilicate glass was taken to be 1.53 and the absorption coefficient $0.1 \mathrm{~cm}^{-1}$ (Siegel, R.; Howell, J. R., Thermal Radiation Heat Transfer. 4th ed.; Taylor \& Francis: New York, 2002). A satisfactory fit was observed in both cases using the LSSE model, thus this model was integrated into the CFD simulations. The radiation emission model described above was essential to explore the effect of radiation intensity on reactor performance.
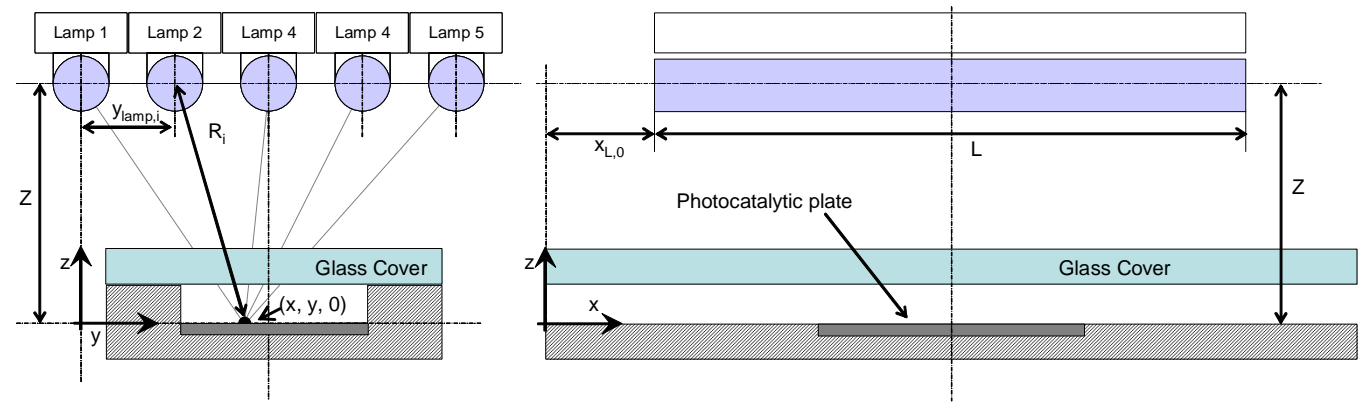

Figure S1. Lamp arrangement showing coordinates of LSSE model. 

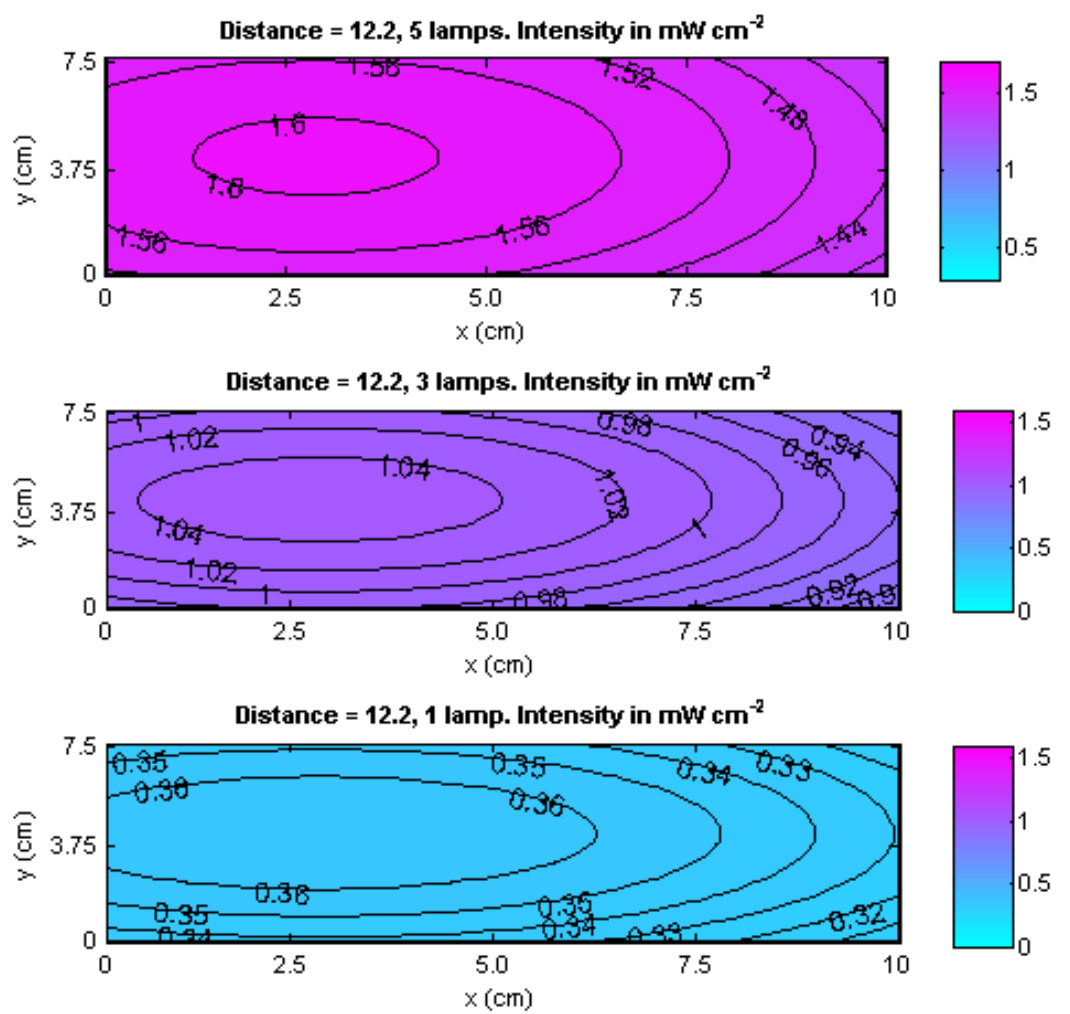

Figure S2. Distribution of radiation intensity on the surface of the catalytic plate for 5,3 and 1 lamp. $Z=0.122 \mathrm{~m},\left\langle\mathrm{l}_{\mathrm{w}}>=73.5 \mathrm{~W} \mathrm{~m}^{-2}\right.$.

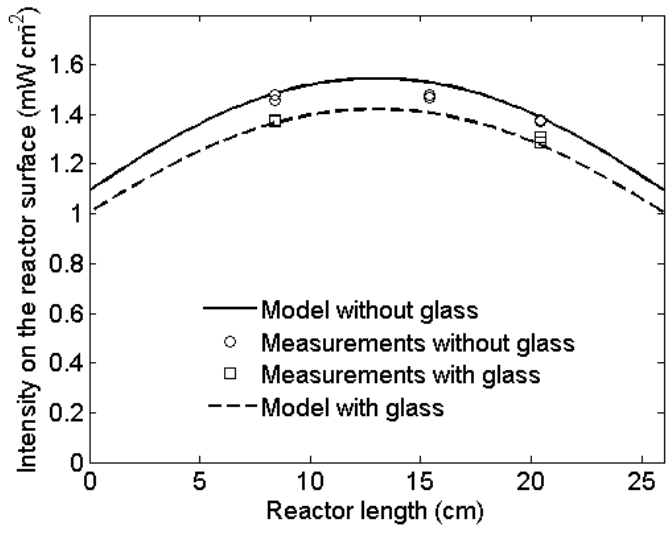

Figure S3. Comparison of model prediction and experimental measurement of radiation intensity at different positions on the catalytic plate, in the presence and in the absence of the borosilicate glass reactor cover. Five UV lamps, Z = $0.122 \mathrm{~m},<\mathrm{I}_{\mathrm{w}}>=73.5 \mathrm{~W} \mathrm{~m}^{-2}$. 
CFD Modeling of Reactor

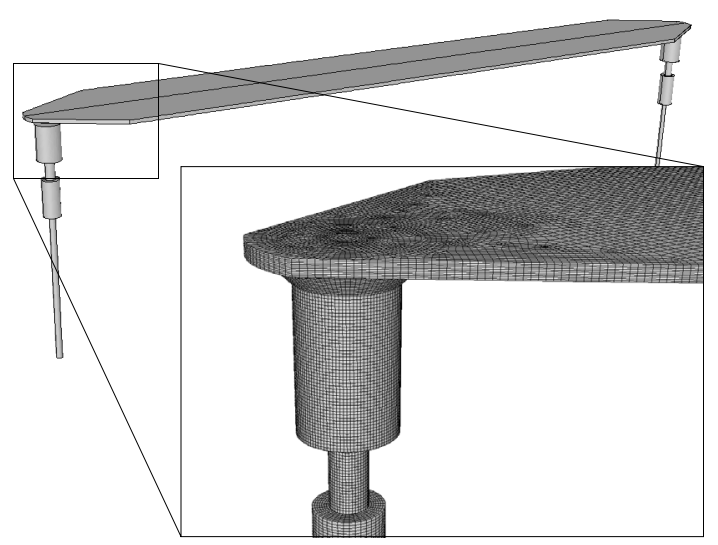

Figure S4. Model of the inlet and outlet pipework and of the reactor, built using the CAD software GAMBIT that is part of the FLUENT software package. The outlet pipework was identical and situated at the opposite end of the model. 
Effect of Inlet and Outlet Pipework Configuration on the Flow Regime Above the Catalyst
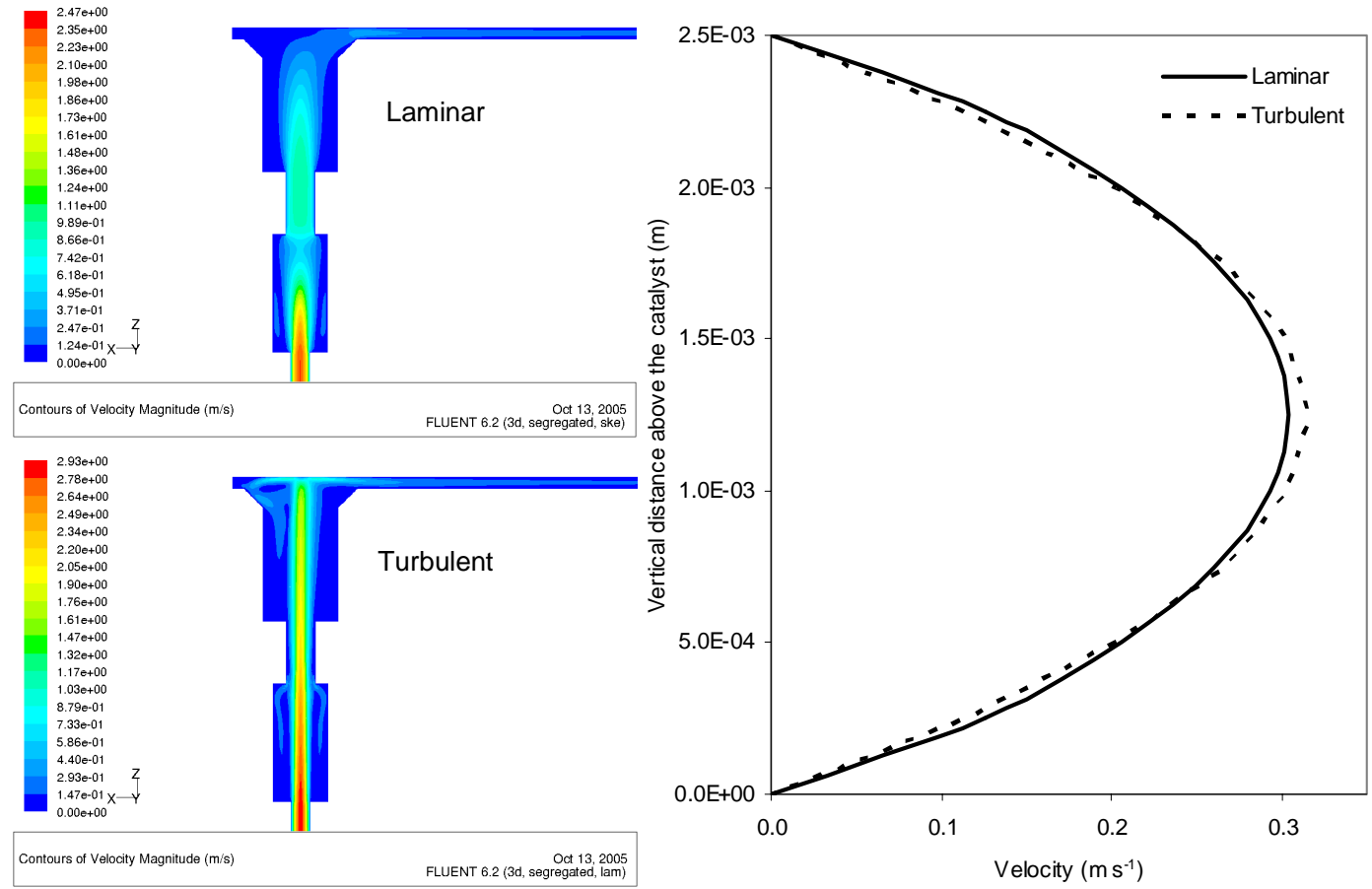

Figure S5. CFD simulations of flow pattern in the reactor using laminar and turbulent flow models. Velocity contour plots for a vertical cross section of the inlet pipework are shown on the left. Velocity profiles above the catalyst are shown on the right. Flow rate $=2.3 \mathrm{~L} \mathrm{~min}^{-1}$. 


\section{Effect of Mass Transfer}

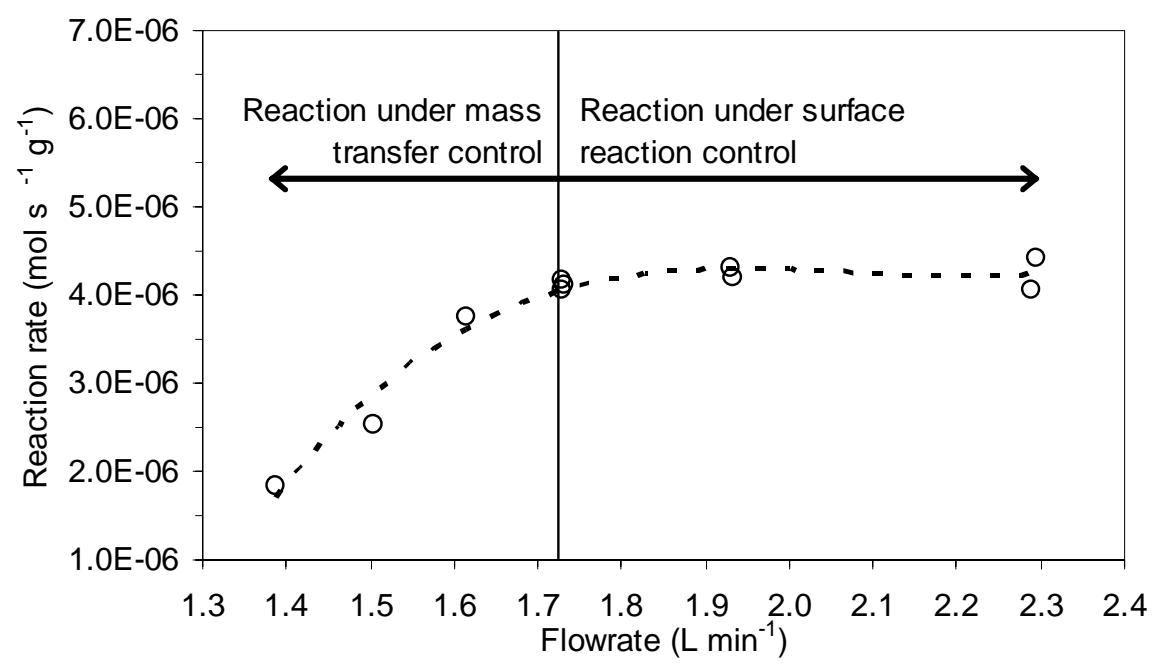

Figure S6. Effect of flow rate on the TCE reaction rate showing mass transfer and kinetic control regions. $C_{T C E, \text { inlet }}=33.2 \mu \mathrm{M},\left\langle I_{W}\right\rangle=73.5 \mathrm{~W} \mathrm{~m}^{-2}$ and five lamps. 


\section{Effect of TCE molar flow rate}

The CFD model predictions using the kinetic parameters in Table 1 were compared against experimental results of TCE oxidation at different molar flow rates in the reaction controlled regime as shown in Figure S6. In these experiments the inlet TCE concentration was kept approximately constant by increasing the TCE feed rate proportionally to the increase in the volumetric flow rate. The model was found to represent the experimental results satisfactorily.

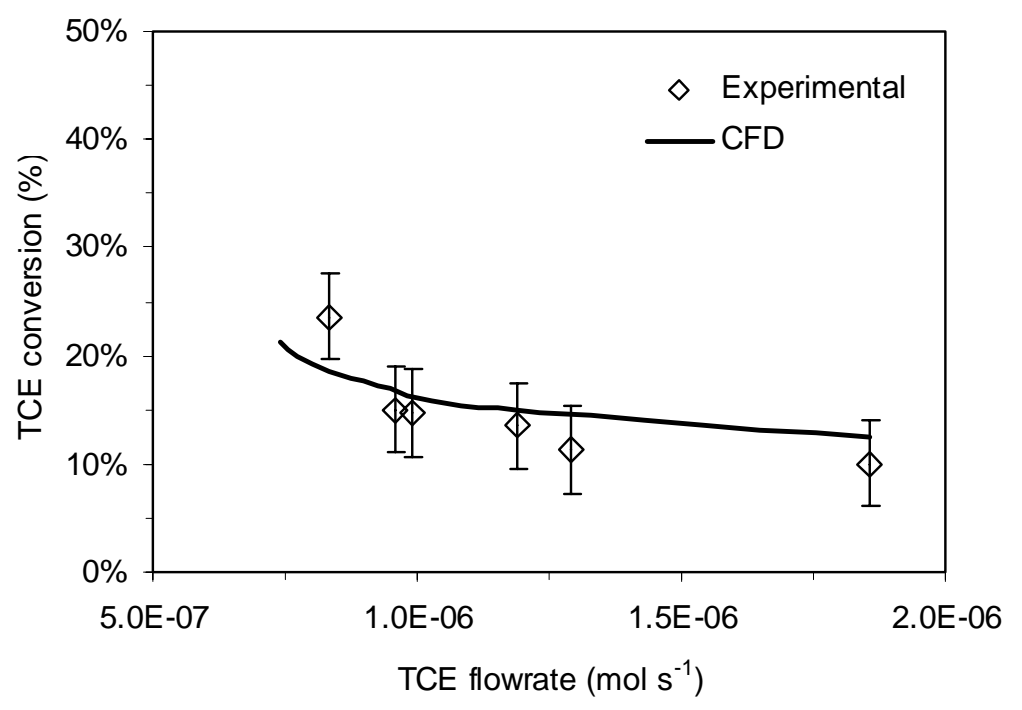

Figure S7. Conversion as a function of TCE molar flow rate. Comparison between experimental and CFD model. $\mathrm{C}_{\mathrm{TCE} \text {,inlet }}$ from 30 to $35 \mu \mathrm{M} .\left\langle\mathrm{I}_{\mathrm{W}}\right\rangle=73.5 \mathrm{~W}$ $\mathbf{m}^{-2}$. 


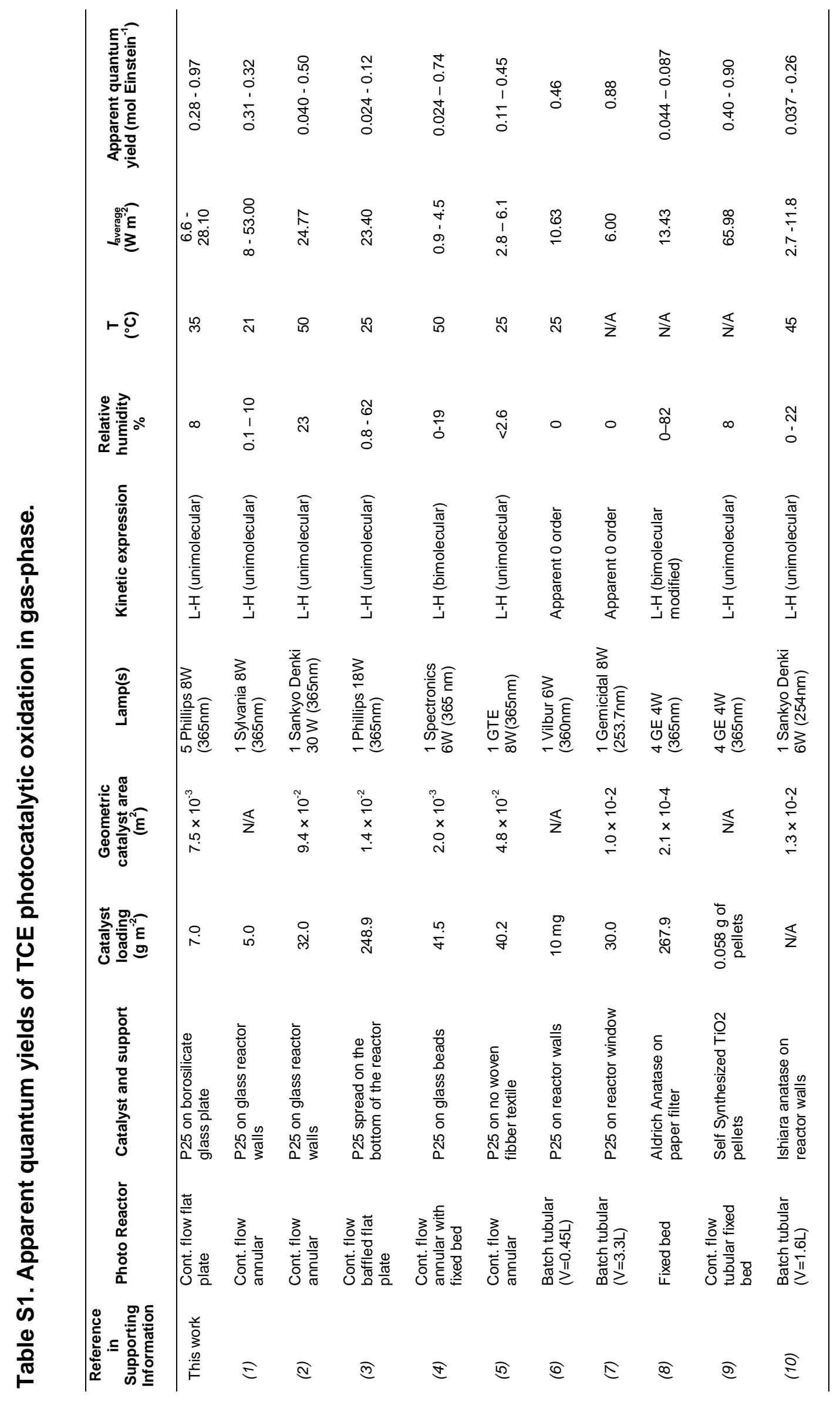

$\stackrel{\infty}{\sim}$ 


\section{REFERENCES IN SUPPORTING INFORMATION}

(1) Jacoby, W. A.; Blake, D. M.; Noble, R. D.; Koval, C. A., Kinetics of the oxidation of trichloroethylene in air via heterogeneous photocatalysis. J. Catal 1995, 157, 87-96.

(2) Alberici, R. M.; Jardim, W. F., Photocatalytic destruction of VOCs in the gasphase using titanium dioxide. Appl. Catal. B: Environ. 1997, 14, 55-68.

(3) Demeestere, K.; Visscher, A. D.; Dewulf, J.; Leeuwen, M. V.; Langenhove, H. V., A new kinetic model for titanium dioxide mediated heterogeneous photocatalytic degradation of trichloroethylene in gas-phase. Appl. Catal. B: Environ. 2004, 54, 261-274.

(4) Wang, K.-H.; Tsai, H.-H.; Hsieh, Y.-H., The kinetics of photocatalytic degradation of trichloroethylene in gas phase over $\mathrm{TiO}_{2}$ supported on glass bead. Appl. Catal. B: Environ. 1998, 17, 313-320.

(5) Ku, Y.; Ma, C.-M.; Shen, Y.-S., Decomposition of gaseous trichloroethylene in a photoreactor with $\mathrm{TiO}_{2}$-coated nonwoven fiber textile. Appl. Catal. B: Environ. 2001, 34, 181-190.

(6) Lichtin, N. N.; Muthusami, A., $\mathrm{TiO}_{2}$-Photocatalyzed Oxidative Degradation of $\mathrm{CH}_{3} \mathrm{CN}, \mathrm{CH}_{3} \mathrm{OH}, \mathrm{C}_{2} \mathrm{HCl}_{3}$, and $\mathrm{CH}_{2} \mathrm{Cl}_{2}$ Supplied as Vapors and in Aqueous Solution under Similar Conditions. Environ. Sci. Technol. 1996, 30, 2014-2020.

(7) Zuo, G. M.; Cheng, Z. X.; Chen, H.; Li, G. W.; Miao, T., Study on photocatalytic degradation of several volatile organic compounds. J. Hazard. Mater. 2006, B128, 158-163.

(8) Dibble, L. A.; Raupp, G. B., Kinetics of the gas-solid heterogeneous photocatalytic oxidation of trichloroethylene by near UV illuminated titanium dioxide. Catal. Lett. 1990, 4, 345-354.

(9) Yamazaki-Nishida, S.; Nagano, K. J.; Phillips, L. A.; Cervera-March, S.; Anderson, M. A., Photocatalytic degradation of trichloroethylene in the gas 
phase using titanium dioxide pellets. J. Photochem. Photobiol. A: Chem. 1993, 70, 95-99.

(10) Kim, S. B.; Hong, S. C., Kinetic study for photocatalytic degradation of volatile organic compounds in air using thin film $\mathrm{TiO}_{2}$ photocatalyst. Appl. Catal. B: Environ. 2002, 35, 305-315. 